\title{
Multidisciplinary assessment of tako tsubo cardiomyopathy: a prospective case study
}

Micael Waldenborg ${ }^{2}$, Mona Soholat ${ }^{3}$, Anders Kähäri $^{4}$, Kent Emilsson ${ }^{2}$ and Ole Fröbert ${ }^{*}$

\begin{abstract}
Background: The cause of tako tsubo cardiomyopathy remains unclear. We used a multidisciplinary approach to investigate if a common pathophysiological denominator could be outlined.

Methods: Within 3 days following symptom presentation and again after 3 months we investigated all patients coming to our institution and diagnosed with tako-tsubo cardiomyopathy. Patients underwent extensive biochemical screening. Left ventricular function was evaluated by echocardiography and contrast-enhanced cardiac magnetic resonance imaging. Cardiac autonomic function was studied by heart rate variability and signal-averaged electrocardiogram and posttraumatic stress and depression were investigated by questionnaires (the Posttraumatic Stress Syndrome 10-Questions Inventory, PTSS-10 and the Montgomery-Åsberg depression rating scale, self rated version, MADRS-S).
\end{abstract}

Results: During 2 years, 13 consecutive patients were included. Markers of myocardial damage and heart failure were slightly to moderately elevated and ejection fraction (echocardiography and MRi) was moderately reduced at hospitalization and improved to normal values in all patients. Signal averaged ECG demonstrated a statistically significant shorter duration of the filtered QRS complex in the acute phase as compared to follow-up. In heart rate variability analysis, SDNN and SDANN were shorter acutely compared to follow-up. Two patients fulfilled criteria for posttraumatic stress syndrome while 7 patients were in the borderline zone. There was a statistically significant inverse correlation between PTSS-10 score and QRS duration in the signal-averaged ECG $(r=-0.66, P=0.01)$.

Conclusions: Patients with tako tsubo cardiomyopathy have altered cardiac autonomic function and a high incidence rate of borderline or definite posttraumatic stress syndrome acutely. This is in line with findings in patients with myocardial infarction and does not allow conclusions on cause and effect.

\section{Background}

Tako-tsubo cardiomyopathy is a rapidly resolving condition of unknown etiology. The typical patient is female, postmenopausal and present with symptoms, electrocardiographic (ECG) and clinical findings suggestive of ST-elevation myocardial infarction. However, coronary arteriography is normal or findings are unrelated to the often severe transient heart failure involving hypokinesia of the left ventricular mid segments and apical ballooning [1].

Tako tsubo cardiomyopathy has been associated with acute emotional stress [2] but this is not obligate [3] and recently it was hypothesized that changes in autonomic control of the cardiovascular system contribute to tako

\footnotetext{
* Correspondence: ole.frobert@orebroll.se

'Department of Cardiology, Örebro University Hospital, Örebro, Sweden

Full list of author information is available at the end of the article
}

tsubo [4]. Other prominent hypotheses on pathophysiology postulate increased release or sensitivity to catecholamines [5] or alteration of $\mathrm{Ca}^{2+}$-handling proteins [6].

The purpose of this study was to offer a comprehensive multidisciplinary approach to all patients presenting with tako tsubo cardiomyopathy in our institution during a 2-year time period in order to better understand and treat the disease. We hypothesized a relation between scores of emotional stress and depression on one side and cardiological, physiological and biochemical measures of disease severity, on the other. In order to address our hypothesis we used an array of conventional cardiac examinations, scores of posttraumatic stress and heart rate variability and signal-averaged ECGs to assess ventricular late potentials at baseline and after 3 months.

\section{Biomed Central}




\section{Methods}

All patients suspected of having ST-elevation myocardial infarction and referred to our institution for acute for coronary arteriography between April 2008 and March 2010 were screened for the study. Criteria for inclusion were in line with the recently published Mayo clinic criteria for tako tsubo cardiomyopathy [7]: hypokinesis, akinesis, or dyskinesis of the left ventricular mid segments with or without apical involvement; absence of obstructive coronary disease or angiographic evidence of acute plaque rupture; new ECG abnormalities (either ST-segment elevation and/or T-wave inversion) and no clinical suspicion of pheochromocytoma or myocarditis. Patient screening and -inclusion was done in the catheterization laboratory following coronary arteriography and left ventriculography. All patients fulfilling the above criteria and giving written informed consent were extensively investigated. We recorded ECGs including assessment of heart rate variability (HRV) and signal-averaged ECGs (SAECG) to measure ventricular late potentials and extensive biochemical profiling including catecholamines was carried out. Left ventricular function was evaluated by echocardiography and cardiac magnetic resonance imaging (MRi) was used for assessment of cardiac function and myocardial viability.

In the acute phase and at the 3-months follow-up HRV was recorded using a battery operated solid-state recorder (DXP1000, Braemar, Eagan, USA) for 24-hour continuous recording of ambulatory ECG data and analyzed using an Aspect Holter system (Danica Biomedical, General Electric, Fairfield, United States). The following time-domain variables were calculated: average $R-R$ interval value calculated from accepted beats (mean RR), standard deviation of all the NN (normal-to-normal) R-R intervals (SDNN), and the baseline width of the minimum square difference triangular interpolation of the highest peak of the histogram of NN intervals (TINN). The long-term HRV was quantified by the standard deviation of all 5-min NN interval mean values (SDANN). NN50 count, that is the number of interval differences of successive normal-to-normal intervals greater than $50 \mathrm{~ms}$ as well as SDSD, that is the mean of standard deviations of normal RR intervals for all 5 -minute segments, were also calculated. Finally, the beatto-beat HRV was estimated by the square root of the mean squared differences of successive NN intervals (RMSSD), and the ratio of NN interval differences of successive NN intervals greater than $50 \mathrm{~ms}$ to the total number of NN intervals (pNN50) [8].

Power spectral analysis was performed on the RRinterval data by means of fast Fourier transformation. The following components were measured: very low frequency (VLF), low frequency (LF), high frequency (HF) and the total power.
The SAECGs were obtained using a Megacart ECG recorder (Siemens-Elema AB, Solna, Sweden). In total, 300 beats were analysed using a noise reduction of 0.3 $\mu \mathrm{V}$ and a correlation of 0.98 . The bipolar $\mathrm{X}, \mathrm{Y}$ and $\mathrm{Z}$ lead system was used. The duration of the filtered QRS-complex (fQRS), the root mean square voltage, that is the RMS-amplitude during the last $40 \mathrm{~ms}$ of the filtered QRS-complex (RMS40) and the low amplitude signal, that is the time during which the amplitude of the filtered QRS-complex last part remains below $40 \mu \mathrm{V}$ (LAS40) [8].

A Vivid 7 ultrasound machine (GE Vingmed Ultrasound A/S, Horten, Norway) equipped with a multi-frequency phased array transducer (M3S, 1.5-4.0 MHz) was used for the echocardiographic examinations and measurements were made after the examination using stored images on an EchoPac workstation (GE Vingmed). The subjects were examined in the left lateral recumbent position. Left ventricular ejection fraction (LVEF) was measured using the biplane Simpson's method

MRi was done with a Philips Achieva 1.5-T scanner. Standard bTFE (balanced Turbo Field Echo) cine-images were acquired as single $8 \mathrm{~mm}$ slice in long-axis, 3-chamber and 4-chamber view. Full ventricular coverage was achieved with 15-20 short-axis slices of $5 \mathrm{~mm}$ thickness. A delayed enhancement protocol was used 15 minutes after intravenous injection of Gadiodiamid $(0.15 \mathrm{mmol} / \mathrm{kg})$ with breath-hold inversion-recovery TFE using 10 slices of $10 \mathrm{~mm}$ thickness in long-axis, 3-chamber and 4-chamber view. About 15 slices were acquired in short-axis to get full ventricular coverage.

Patients answered questionnaires on posttraumatic stress (the Posttraumatic Stress Syndrome 10-Questions Inventory, PTSS-10) and depression (MontgomeryÅsberg depression rating scale, self-rated version, MADRS-S). The PTSS-10 is a self-administered questionnaire that measures the current presence of specific posttraumatic stress symptoms. Patients are asked to rate the presence of each symptom during the past 7 days and its severity on a scale from 1 (never) to 7 (always). A total score $>35$ is associated with a high probability that the diagnostic criteria for post-traumatic stress disorder are fulfilled and a score between 27 and 35 is borderline [9]. The MADRS-S is an observer rating scale that records 10 depression items between 0 and 6 , according to their intensity. A score $>34$ is associated with major depression on the MADRS-S while 20-33 is considered borderline [10]. Both rating scales are available in Swedish.

All blood sampling was done in the morning after admission to the hospital, echocardiography within 24 hours and all other testing within 72 hours after admission. Most tests were repeated at a 3 months clinical control. The study protocol was approved by the Regional Ethical Committee in Uppsala, Sweden. 
Statistical analysis was performed using SigmaStat 3.5 software (Systat, San Jose, Ca). Most variables were not normally distributed and we therefore used the Wilcoxon signed rank test for pairwise comparisons and Spearman's rank correlation coefficient for nonparametric assessment of statistical dependence between two variables. Differences were considered statistically significant when $\mathrm{P}<0.05$.

\section{Results}

In the 2-year study period, between April 2008 and March 2010, 15 patients, all women, fulfilled criteria for inclusion. Two patients declined participation and 13 patients were included in the study (Table 1). All patients were treated with beta adrenergic receptor antagonists and angiotensin-converting-enzyme inhibitors between the acute phase and the 3-months follow-up.

At arrival in the hospital, 9 patients had ST-segment elevation in two or more leads, 3 patients had poor R-wave progression and concomitant $\mathrm{T}$-wave changes in the precordial leads and one patient had precordial ST-segment depressions. Markers of myocardial damage and heart failure were slightly to moderately elevated in all patients (Table 2). Catecholamines and thyroid parameters were normal and did not change from initial hospitalization to the 3 -months control visit (although noradrenalin was borderline significant). Average cholesterol values were slightly above reference level and did also not change during follow-up (Table 2).

Of the 13 study patients, in 11 the left ventriculogram demonstrated apical ballooning and hypercontractile basal segments. In one patient a left ventriculogram was not performed but acute echocardiography demonstrated a similar pattern. In one patient only did the ventriculogram demonstrate an inverse pattern - mid-ventricular ballooning and apical akinesia. Ejection fraction was moderately reduced at hospitalization as demonstrated by both echocardiography and MRi and improved to normal values for all patients (Table 3 ). MRi could not be done in four patients (one had pacemaker, one elevated serum creatinine and two patients suffered from claustrophobia) but in

Table 1 Baseline data of the patients $(n=13)$

\begin{tabular}{cl}
\hline Variable & Median or number \\
\hline Age, years & $70(69-74)$ \\
Women, $\mathrm{n}(\%)$ & $13(100)$ \\
Height, cm & $158(152-163)$ \\
Weight, kg & $65(55-80)$ \\
Diabetes mellitus, n (\%) & $0(0)$ \\
Current smoker, $\mathrm{n}(\%)$ & $1(8)$ \\
Family history of IHD, n (\%) & $3(23)$ \\
Definite trauma within 2 weeks, n (\%) & $6(46)$ \\
\hline
\end{tabular}

Values are median (interquartile range) or number (percentage). none of the 9 patients undergoing MRi did late gadolinium enhancement demonstrate signs of myocardial necrosis.

Signal averaged ECG recordings demonstrated a statistically significant shorter duration of the filtered QRS complex in the acute phase as compared to 3-months follow-up (despite the fact that the median values were almost identical, Table 4). Also in the acute phase there was a trend towards reduced root mean square voltage of the signal in the last $40 \mathrm{~ms}$ of the QRS complex (RMS 40) and of the low amplitude signal under $40 \mu \mathrm{v}$ (LAS 40), but these parameters did not reach statistical significance.

In HRV analysis, two time domain parameters came out statistically significant: standard deviation of the normal-to-normal interval (SDNN) and the standard deviation of sequential 5-min heart period mean values (SDANN) which were both shorter acutely as compared to three months follow-up (table 4). No other HRV parameter differed between the two time points.

Only one patient had a history of mental illness. This patient had been diagnosed with manio-depressive psychosis years before being admitted with tako tsubo. Her manio-depressive disease was considered stable at admittance and she scored 31 and 33 in the PTSS-10 scale (borderline) while the score was 13 on MADRS-S scale (no depression). At admission, 2 patients fulfilled criteria for posttraumatic stress syndrome while 7 patients were in the borderline zone. At 3 months follow-up one patient still fulfilled criteria for posttraumatic stress syndrome while 4 had borderline scores (Figure 1). We tested associations between PTSS-10 score and major variables of cardiac function (EF, NT pro-brain natriuretic peptide, TnI, adrenalin, noradrenalin, late potentials in the ECG and heart rate variability parameters). There was a statistically significant inverse correlation between PTSS-10 score and QRS duration in the signal-averaged ECG ( $\mathrm{r}=$ $-0.66, P=0.01$, Figure 2). Using the MADRS-S score one patient was in the borderline zone for depression acutely while all patients had scores within the normal range at follow-up. For the entire patient group there were no statistically significant differences in rating scale scores (PTSS-10 or MADRS-S) in the acute phase compared to 3-months follow-up (Table 5).

\section{Discussion}

The main findings of this study on consecutive patients with tako tsubo cardiomyopathy were that in addition to clinical and laboratory indices of heart failure, cardiac autonomic function was altered and we found a relation between emotional stress and QRS duration.

Although the signal-averaged ECG values were within the limits of previously published normal values [11] we did find a significantly shorter duration of the filtered QRS in the acute phase compared to recovery. 
Table 2 Biochemical parameters and findings

\begin{tabular}{|c|c|c|c|c|}
\hline Variable & Initial findings & 48 hours & 3 months & $\mathbf{P}$ \\
\hline Troponin I (mg/L) & $2.70(0.45-2.90)$ & $0.42(0.17-0.76)$ & Not measured & $<0.001$ \\
\hline Creatine kinase $\mu \mathrm{kat} / \mathrm{L}$ & $2.55(1.58-3.15)$ & $1.50(1.00-1.90)$ & Not measured & 0.03 \\
\hline Creatin kinase MB $(\mu \mathrm{g} / \mathrm{L})$ & $11.60(5.35-22.33)$ & $4.40(2.80-5.10)$ & Not measured & 0.002 \\
\hline NT pro-brain natriuretic peptide (ng/L) & $389(228-447)$ & $313(275-702)$ & Not measured & 0.32 \\
\hline High-sensitivity C-reactive protein (mg/L) & $4.10(2.90-6.70)$ & $10.50(7.10-19.60)$ & Not measured & 0.02 \\
\hline Thyroid-stimulating hormone (mIU/L) & $1.20(0.68-1.70)$ & Not measured & $1.10(0.90-1.50)$ & 0.97 \\
\hline Thyroxine $(\mathrm{pmol} / \mathrm{L})$ & $14.30(13.60-15.50)$ & Not measured & $14.00(13.30-15.00)$ & 0.57 \\
\hline Cholesterol (mmol/L) & $6.10(5.30-6.60)$ & Not measured & $5.40(4.40-6.00)$ & 0.57 \\
\hline Low-density lipoprotein cholesterol (mmol/L) & $3.60(2.70-4.40)$ & Not measured & $3.00(2.10-3.50)$ & 0.52 \\
\hline High-density lipoprotein cholesterol (mmol/L) & $1.50(1.20-1.50)$ & Not measured & $1.40(1.30-1.60)$ & 0.18 \\
\hline Triglyceride $(\mathrm{mmol} / \mathrm{L})$ & $1.60(1.20-2.20)$ & Not measured & $1.50(1.20-2.40)$ & 0.41 \\
\hline Plasma adrenalin $(\mathrm{nmol} / \mathrm{l}) *$ & $0.30(0.30-0.40)$ & Not measured & $0.30(0.30-0.30)$ & 1.00 \\
\hline Plasma noradrenalin $(\mathrm{nmol} / \mathrm{l}) *$ & $3.70(2.60-5.50)$ & Not measured & $3.30(2.50-3.40)$ & 0.06 \\
\hline
\end{tabular}

Values are median (interquartile range) or number (percentage).

*Complete data from 7 patients only.

Filtered QRS duration is under influence of autonomic tone [12] but is it is not likely that the beta blocker treatment introduced in all patients influenced this parameter [13]. Filtered QRS duration has been associated with ventricular arrhythmias in non-ischemic heart failure [14], with ventricular asynchrony in chronic heart failure patients [15] and is reduced in healthy subjects to mental stress [16]. The finding of reduced filtered QRS duration acutely despite reduced LVEF and presumable susceptibility to ventricular arrhythmias in tako tsubo patients [17] in our view supports that emotional stress had more impact on this parameter than LV dysfunction.

HRV measured shortly after coming to the hospital with acute symptoms and again after recovery at 3 months demonstrated lower values of the time domain parameters SDNN and SDANN acutely. A German group recently made similar findings on HRV parameters [18] and the same group also published data that apical and midventricular variants of tako tsubo patients differ in some key HRV variables [19]. SDANN reflects both a sympathetic and parasympathetic heart rate modulation while a depressed SDANN is thought to indicate a relative sympathetic dominance [20]. In studies of heart failure patients SDNN and SDANN have been found to correlate inversely with plasma endothelin-1 [21] and noradrenaline [22] while acute pain had no effect on SDNN in a study of healthy male persons [23]. In a study of 138 patients in the early phase of acute myocardial infarction Doulalas et al. found similar SDNN values as we did in the present study (mean SDNN: $86 \pm 35$, SDANN: $74 \pm 29$ ) [24]. Emotional stress has been seen as a major contributor to causing tako tsubo cardiomyopathy and emotional stress also affects HRV. In one study a mental stress task significantly reduced SDNN index in patients after myocardial infarction [25]. In this study there was a statistically insignificant trend towards reduced SDNN index in the acute phase. Taken together, these different findings make it difficult to draw a firm conclusion on the acute SDNN reductions in our study; they might be a product of temporary heart failure or caused by emotional stress or even a blend of the two.

Posttraumatic stress is not uncommon in patients following myocardial infarction and was reported in $22 \%$ of patients in one study [26]. Thus, we cannot conclude that our findings of posttraumatic stress syndrome in two patients and borderline posttraumatic stress syndrome in another 7 support that psychological stress has a say in causing tako-tsubo in these patients. As with myocardial infarction patients, psychological stress in patients with tako tsubo cardiomyopathy can be secondary to chest pain and acute heart failure. It might have been more

Table 3 Data of left ventricular function

\begin{tabular}{|c|c|c|c|}
\hline Variable & Initial findings & 3 months & $\mathbf{P}$ \\
\hline Ejection fraction, echocardiography (Simpsons method), \% & $51(46-55)$ & $65(59-66)$ & 0.003 \\
\hline Ejection fraction, magnetic resonnance imaging, \%, * & $50(54-62)$ & $73(66-74)$ & 0.02 \\
\hline End-diastolic volume, magnetic resonnance imaging, ml, * & $134(120-143)$ & $129(117-140)$ & 0.06 \\
\hline End-diastolic diameter, magnetic resonnance imaging, $\mathrm{mm}^{*}$ & $41(46-51)$ & $44(44-46)$ & 0.56 \\
\hline
\end{tabular}

Values are median (interquartile range).

*Complete data from 9 patients only. 
Table 4 Data of late potentials and heart rate variability

\begin{tabular}{clll}
\hline Late potentials & Initial findings & $\mathbf{3}$ months & $\mathbf{P}$ \\
\hline fQRS, ms & $104(96-108)$ & $103(99-113)$ & 0.01 \\
RMS 40, mV & $31(16-38)$ & $30(10-34)$ & 0.13 \\
LAS 40, ms & $31(27-40)$ & $35(30-46)$ & 0.06 \\
\hline Heart rate variability & Initial findings & $\mathbf{3}$ months & $\mathbf{P}$ \\
\hline Total, ms ${ }^{2}$ & $1300(764-2241)$ & $1881(1276-2065)$ & 0.24 \\
VLF, ms & $929(563-1463)$ & $948(875-1352)$ & 0.34 \\
LF, ms $^{2}$ & $207(154-469)$ & $315(186-558)$ & 0.17 \\
HF, ms & $155(60-216)$ & $132(109-519)$ & 0.59 \\
SDNN, ms & $91(75-126)$ & $114(105-146)$ & 0.008 \\
SDANN, ms & $78(63-111)$ & $103(89-138)$ & 0.008 \\
RMSSD, ms & $28(21-35)$ & $24(22-41)$ & 0.59 \\
SDNN index, ms & $41(31-51)$ & $47(40-49)$ & 0.17 \\
SDSD, ms & $28(21-35)$ & $24(22-41)$ & 0.59 \\
NN50 count & $4157(967-5085)$ & $2913(1448-12500)$ & 0.41 \\
pNN50, \% & $3.96(2.38-6.39)$ & $3.02(1.66-14.47)$ & 0.50 \\
TINN, ms & $390(290-600)$ & $480(400-610)$ & 0.06 \\
\hline
\end{tabular}

Values are median (interquartile range) or number (percentage). Abbreviations are: $\mathrm{fQRS}=$ duration of filtered QRS complex. RMS $40=$ Root mean square voltage of signal in last $40 \mathrm{~ms}$ of QRS. LAS $40=$ Low amplitude signal under $40 \mu \mathrm{v} . \mathrm{VLF}=$ very low frequency, $L F=$ low frequency, HF = high frequency. SDNN = standard deviation of the normal-to-normal interval. SDANN = standard deviation of sequential 5-min heart period mean values. RMSSD = mean squared differences of successive normal-to-normal intervals. SDNN index = mean of standard deviations of normal RR intervals for all 5-minute segments. SDSD = standard deviation of successive differences. NN50 count = the number of interval differences of successive normal-to-normal intervals greater than $50 \mathrm{~ms}$. pNN50 = percentage of adjacent normal-to-normal interval differences $>50 \mathrm{~ms}$. TINN = triangular interpolation of RR intervals.

relevant if the patients had been examined for acute stress disorder. However, the information collected on the psychiatric surveys did not provide enough information to determine this.

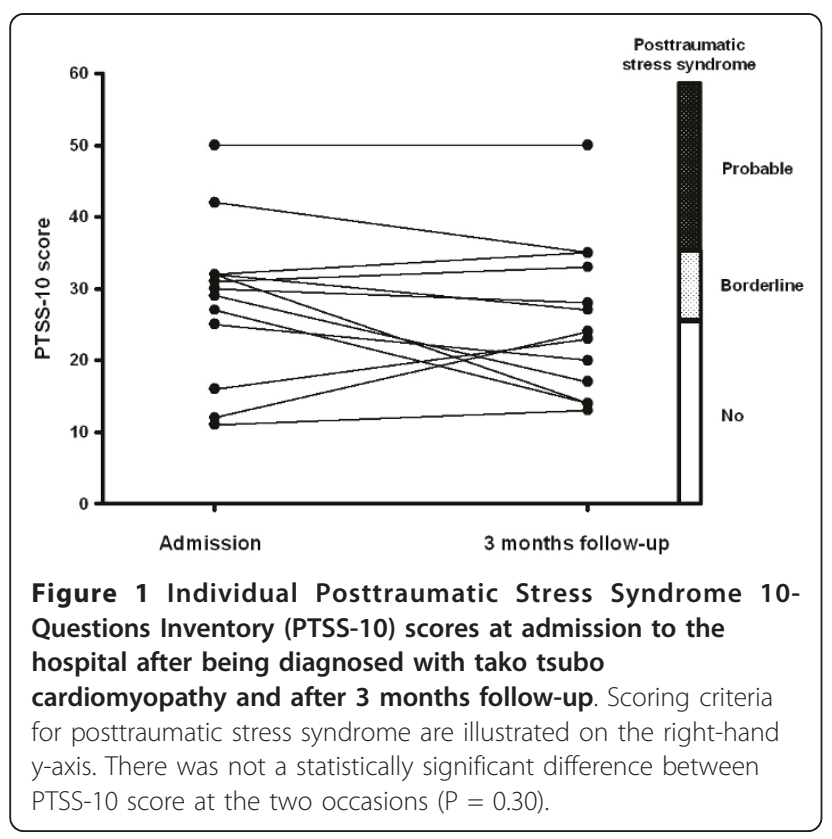

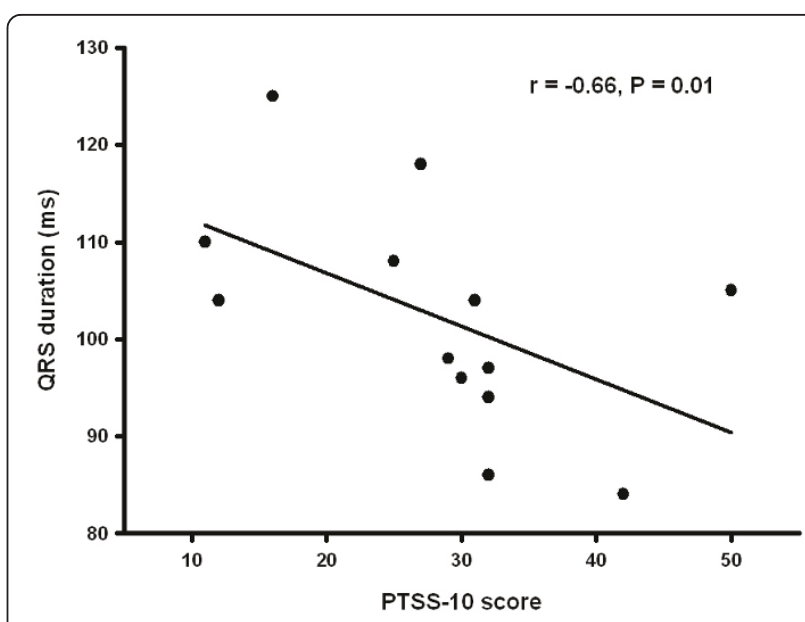

Figure 2 Inverse correlation between Individual Posttraumatic Stress Syndrome 10-Questions Inventory (PTSS-10) scores at admission to the hospital after being diagnosed with tako tsubo cardiomyopathy and QRS duration in the signalaveraged ECG.

Biochemical markers of myocardial damage were slightly to moderately elevated in the present study and this corresponds with previous findings in patients with tako tsubo cardiomyopathy [2,27]. In contrast with an earlier study [5] we did not find elevation in plasma catecholamines. However, we took only one blood sample and the routine use of intravenous diazepam before catheterization in our institution might have reduced catecholamine excretion [28]. Furthermore, ejection fractions as measured by echocardiography (recently published in detail elsewhere [29]) and MRi were reduced only moderately compared with findings by others $[2,27]$. We have no definite explanation to this difference; the timing of examinations in our study was identical to the previous ones and in line with these studies ejection fraction improved to normal.

Although the bulk of literature on tako tsubo cardiomyopathy is confined to case reports this study is limited by its size and by the fact that data come from a single center. Only a subset of patients was examined by MRi and had plasma catecholamine investigations but complete data on both parameters are unlikely to have changed our overall conclusion. Nevertheless, to our knowledge, this study is one of the most comprehensive and detailed investigations of patients with tako tsubo

Table 5 Results of stress and depression rating scales

\begin{tabular}{llll}
\hline \multicolumn{1}{c}{ Rating scale } & Initial findings & $\mathbf{3}$ months follow-up & $\mathbf{P}$ \\
\hline PTSS 10 questionnaire & $30(25-32)$ & $24(17-33)$ & 0.3 \\
MADRS questionnaire & $8(4-10)$ & $10(5.5-11)$ & 0.49 \\
\hline
\end{tabular}

Values are median (interquartile range). Abbreviations are: PTSS $10=$ Posttraumatic Stress Syndrome 10-Questions Inventory. MADRS = Montgomery-Åsberg depression rating scale. 
cardiomyopathy and the novel combined assessment of scores of emotional stress and HRV parameters seems clinically relevant and might inspire future studies of this disease.

\section{Conclusions}

We conclude that patients with tako tsubo cardiomyopathy, in addition to clinical and laboratory indices of heart failure, have altered cardiac autonomic function and a high prevalence of borderline or definite posttraumatic stress syndrome. This is in line with findings in patients with myocardial infarction and does not allow conclusions on cause and effect in tako tsubo cardiomyopathy. In our opinion terms such as "stress-induced cardiomyopathy" or "broken heart syndrome" for this condition should be avoided.

\section{Acknowledgements}

We thank Jan Myrin, MSci for conducting heart rate variability examinations and Karin Loiske MSci for doing echocardiography.

\section{Author details}

'Department of Cardiology, Örebro University Hospital, Örebro, Sweden. ${ }^{2}$ Department of Clinical Physiology, Örebro University Hospital, Örebro, Sweden. ${ }^{3}$ Department of Psychiatry, Örebro University Hospital, Örebro, Sweden. ${ }^{4}$ Department of Radiology, Orebro University Hospital, Orebro, Sweden.

\section{Authors' contributions}

MW performed echocardiography, data management and analysis. MS participated in the design of the study and patient inclusion. AK designed the MRI investigations and did the MRI analyses. KE participated in study design, echocardiography and helped to draft the manuscript. OF designed the study, performed coronary angiographies, statistical analyses and drafted the manuscript. All authors read and approved the final manuscript.

\section{Competing interests}

The authors declare that they have no competing interests.

Received: 9 December 2010 Accepted: 9 April 2011

Published: 9 April 2011

\section{References}

1. Kurisu S, Sato H, Kawagoe T, Ishihara M, Shimatani Y, Nishioka K, et al: Takotsubo-like left ventricular dysfunction with ST-segment elevation: a novel cardiac syndrome mimicking acute myocardial infarction. Am Heart J 2002, 143:448-455.

2. Sharkey SW, Lesser JR, Zenovich AG, Maron MS, Lindberg J, Longe TF, et al: Acute and reversible cardiomyopathy provoked by stress in women from the United States. Circulation 2005, 111:472-479.

3. Sharkey SW, Windenburg DC, Lesser JR, Maron MS, Hauser RG, Lesser JN, et al: Natural history and expansive clinical profile of stress (tako-tsubo) cardiomyopathy. J Am Coll Cardiol 2010, 55:333-341.

4. Nguyen SB, Cevik C, Otahbachi M, Kumar A, Jenkins LA, Nugent K: Do comorbid psychiatric disorders contribute to the pathogenesis of takotsubo syndrome? A review of pathogenesis. Congest Heart Fail 2009, 15:31-34.

5. Wittstein IS, Thiemann DR, Lima JA, Baughman KL, Schulman SP, Gerstenblith $G$, et al: Neurohumoral features of myocardial stunning due to sudden emotional stress. N Engl J Med 2005, 352:539-548.

6. Nef HM, Mollmann H, Troidl C, Kostin S, Voss S, Hilpert P, et al: Abnormalities in intracellular $\mathrm{Ca} 2+$ regulation contribute to the pathomechanism of Tako-Tsubo cardiomyopathy. Eur Heart J 2009, 30:2155-2164.
7. Madhavan M, Prasad A: Proposed Mayo Clinic criteria for the diagnosis of Tako-Tsubo cardiomyopathy and long-term prognosis. Herz 2010.

8. Heart rate variability: standards of measurement, physiological interpretation and clinical use. Task Force of the European Society of Cardiology and the North American Society of Pacing and Electrophysiology. Circulation 1996, 93:1043-1065.

9. Weisaeth L: Torture of a Norwegian ship's crew. The torture, stress reactions and psychiatric after-effects. Acta Psychiatr Scand Suppl 1989, 355:63-72.

10. Montgomery SA, Asberg M: A new depression scale designed to be sensitive to change. Br J Psychiatry 1979, 134:382-389.

11. Timmermans C, Ector H, Haisty KW, Hammill SC, Kienzle MG, Ozawa Y, et al: Signal-averaged ECG parameters in cardiac normals using Frank lead system and Fourier transform filter and gender specific differences: a multicenter study. Pacing Clin Electrophysiol 1994, 17:303-311.

12. Christiansen EH, Frobert O: Effect of parasympathetic blockade on the signal-averaged electrocardiogram. Clin Auton Res 1998, 8:165-171.

13. Goldberger JJ, Ahmed MW, Parker MA, Kadish AH: Assessment of effects of autonomic stimulation and blockade on the signal-averaged electrocardiogram. Circulation 1994, 89:1656-1664.

14. Poll DS, Marchlinski FE, Falcone RA, Josephson ME, Simson MB: Abnormal signal-averaged electrocardiograms in patients with nonischemic congestive cardiomyopathy: relationship to sustained ventricular tachyarrhythmias. Circulation 1985, 72:1308-1313.

15. Tahara T, Sogou T, Suezawa C, Matsubara H, Tada N, Tsushima S, et al: Filtered QRS duration on signal-averaged electrocardiography correlates with ventricular dyssynchrony assessed by tissue Doppler imaging in patients with reduced ventricular ejection fraction. J Electrocardiol 2010, 43:48-53.

16. Folino AF, Buja G, Turrini P, Oselladore L, Nava A: The effects of sympathetic stimulation induced by mental stress on signal-averaged electrocardiogram. Int J Cardiol 1995, 48:279-285.

17. Mahfoud F, Ukena C, Kindermann M, Mewis C, Bohm M: The vulnerable myocardium: long-QT syndrome in Tako-Tsubo cardiomyopathy. Clin Res Cardiol 2009, 98:409-411.

18. Ortak J, Khattab K, Barantke M, Wiegand UK, Bansch D, Ince H, et al: Evolution of cardiac autonomic nervous activity indices in patients presenting with transient left ventricular apical ballooning. Pacing Clin Electrophysiol 2009, 32(Suppl 1):S21-S25.

19. Bonnemeier H, Demming T, Weidtmann B, Ortak J, Burgdorf C, Reppel M, et al: Differential heart rate dynamics in transient left ventricular apical and midventricular ballooning. Heart Rhythm 2010, 7:1825-1832.

20. Lombardi F: Clinical implications of present physiological understanding of HRV components. Card Electrophysiol Rev 2002, 6:245-249.

21. Aronson D, Mittleman MA, Burger AJ: Role of endothelin in modulation of heart rate variability in patients with decompensated heart failure. Pacing Clin Electrophysiol 2001, 24:1607-1615.

22. Burger AJ, Aronson D: Activity of the neurohormonal system and its relationship to autonomic abnormalities in decompensated heart failure. J Card Fail 2001, 7:122-128.

23. Terkelsen AJ, Molgaard H, Hansen J, Andersen OK, Jensen TS: Acute pain increases heart rate: differential mechanisms during rest and mental stress. Auton Neurosci 2005, 121:101-109.

24. Doulalas AD, Flather MD, Pipilis A, Campbell S, Studart F, Rizos IK, et al: Evolutionary pattern and prognostic importance of heart rate variability during the early phase of acute myocardial infarction. Int I Cardiol 2001, 77:169-179.

25. Tuininga YS, Crijns HJ, Brouwer J, Van Den Berg MP, Man iV, Mulder G, et al: Evaluation of importance of central effects of atenolol and metoprolol measured by heart rate variability during mental performance tasks, physical exercise, and daily life in stable postinfarct patients. Circulation 1995, 92:3415-3423.

26. Pedersen SS, Middel B, Larsen ML: Posttraumatic stress disorder in firsttime myocardial infarction patients. Heart Lung 2003, 32:300-307.

27. Singh NK, Rumman S, Mikell FL, Nallamothu N, Rangaswamy C: Stress cardiomyopathy: clinical and ventriculographic characteristics in 107 North American subjects. Int J Cardiol 2010, 141:297-303.

28. Liu WS, Bidwal AV, Lunn JK, Stanley TH: Urine catecholamine excretion after large doses of fentanyl, fentanyl and diazepam and fentanyl, diazepam and pancuronium. Can Anaesth Soc J 1977, 24:371-379. 
29. Loiske K, Waldenborg M, Frobert O, Rask P, Emilsson K: Left and right ventricular systolic long-axis function and diastolic function in patients with takotsubo cardiomyopathy. Clin Physiol Funct Imaging 2010.

\section{Pre-publication history}

The pre-publication history for this paper can be accessed here: http://www.biomedcentral.com/1471-2261/11/14/prepub

doi:10.1186/1471-2261-11-14

Cite this article as: Waldenborg et al:: Multidisciplinary assessment of tako tsubo cardiomyopathy: a prospective case study. BMC

Cardiovascular Disorders 2011 11:14.

Submit your next manuscript to BioMed Central and take full advantage of:

- Convenient online submission

- Thorough peer review

- No space constraints or color figure charges

- Immediate publication on acceptance

- Inclusion in PubMed, CAS, Scopus and Google Scholar

- Research which is freely available for redistribution

Submit your manuscript at www.biomedcentral.com/submit 